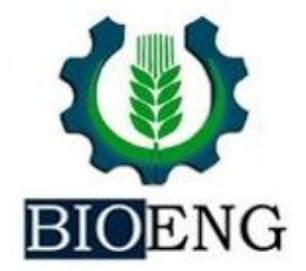

\title{
VARIABILIDADE ESPACIAL DA QUALIDADE DO CAFÉ CEREJA
}

\author{
M. M. Baesso*, F. T. P. Koja, A. Chi Lin Sun, A. J. Modolo, \\ F. L. Caneppele
}

USP - Universidade de São Paulo, Faculdade de Zootecnia e Engenharia de Alimentos, Campus Fernando Costa, Pirassununga, SP, Brasil

Article history: Received 21 February 2019; Received in revised form 03 May 2019; Accepted 14 May 2019; Available online 24 June 2019.

\section{RESUMO}

A qualidade do café pode variar espacialmente em uma mesma área de acordo com as interações do cenário ambiental e genético apresentado à cultura. Essa variabilidade espacial pode ser decisiva para o produtor, visto que o valor comercial do café é influenciado pela sua qualidade. Uma alternativa viável para supor a qualidade do café é a partir do teor de sólidos solúveis (graus brix) do fruto. Dessa forma, foi analisado a variabilidade espacial do teor de sólidos no café conilon, colhido no estágio cereja, visando determinar as zonas de manejo em termos de qualidade. $\mathrm{O}$ método foi desenvolvido em uma propriedade de 2 ha, no município de Ribeirão Corrente, no norte do Estado de São Paulo. Foi realizada uma amostragem georreferenciada do café cereja e aferição dos valores de brix, por meio de um refratômetro portátil. A análise dos dados foi feita pelos métodos de dendrograma e "K means", definindo três classes para os valores de brix, abaixo de 16,5, entre 16,6 e 19,7 e superiores a 19,8. Em seguida obteve-se um mapa que indica a variabilidade espacial dos graus brix dos frutos de café cereja e conclui-se que há uma pequena área da propriedade com maior potencial para a produção de um café de qualidade superior. Indicando, a oportunidade de emprego de um manejo específico que privilegie a comercialização dos frutos produzidos nesse local.

Palavras-chave: sensoriamento remoto, graus brix, cartas de controle.

\section{SPATIAL VARIABILITY OF CHERRY COFFEE QUALITY}

\begin{abstract}
The quality of the coffee may vary spatially in the same area according to the interaction of environmental and genetic background presented to the culture. This spatial variability can be decisive for the producer, since the commercial value of coffee is influenced by its quality. A viable alternative to assume the quality of the coffee is from the soluble solids content (degrees brix) of the fruit. In this way, the spatial variability of solids content in conilon coffee harvested at the cherry stage was analyzed in order to determine management zones in terms of quality. The method was developed on a 2 ha property in the municipality of Ribeirão Corrente, in the north of the State of São Paulo. A georeferenced sample of the cherry coffee was carried out and the values of brix were measured by means of a portable refractometer. Data analysis was performed using dendrogram and "K means" methods, defining three classes for brix values, below 16.5, between 16.6 and 19.7 and above 19.8. A
\end{abstract}

\footnotetext{
*baesso@usp.br
} 
map indicating the spatial variability of the brix grades of the cherry coffee fruits was obtained and it was concluded that there is a small area of the property with greater potential to produce of a higher quality coffee. Indicating, the opportunity to use a specific management that privileges the commercialization of the fruits produced in that place.

Keywords: remote sensing, brix degrees, control charts.

\section{INTRODUÇÃO}

O café, proveniente das regiões montanhosas africanas da Abissínia, foi difundido pelo mundo e ingressou no Brasil no século XVIII, tornando-se uma das commodities mais importantes do país. Atualmente, o Brasil é o maior produtor e exportador mundial de café e, na safra de 2015, colheu cerca de 44,3 milhões de sacas de café (CONAB, 2015). Informações que reiteram a representatividade econômica e a importância social deste cultivo.

Apesar da significativa participação no mercado cafeeiro mundial, os produtores nacionais apresentam dificuldades para oferecer o café no mercado externo de maneira competitiva, em virtude da concorrência por preço e qualidade com outros países produtores (ABIC, 2015). Isto é, a realização da gestão dos fatores de produção de maneira específica, contemplando sua variabilidade espacial, pode auxiliar na identificação de áreas com potencial para produção de grãos de melhor qualidade (QUEIROZ et al., 2004).

Para avaliar essa variabilidade espacial propõe-se a confecção de mapas por georreferenciamento que permitam a associação de atributos do solo (como textura e fertilidade), a localização geográfica, as condições climáticas e a forma de como as operações agrícolas são

\section{MATERIAL E MÉTODOS}

O trabalho foi realizado no norte do estado de São Paulo, no município de Ribeirão Corrente, localizado conduzidas, aferindo também a produtividade, por meio de indicadores de maturidade e palatabilidade do fruto obtidos através dos valores de brix. (SILVA \& LIMA, 2014).

Dessa forma, o processo de georreferenciamento sucede-se a partir da definição de zonas de manejo ou subregiões que se dispõem de características homogêneas (como dimensão da propriedade, relevo, clima local, profundidade) e, consequentemente, devem receber um tratamento comum, principalmente em relação a fatores que interferem diretamente na produtividade da cultura (FERRAZ, et al., 2014).

A determinação do teor de sólidos solúveis (grau brix) é realizada através de um refratômetro digital ajustado a uma escala numérica de índice de refração, desempenhando um recurso viável para a predição da qualidade de bebida, uma vez que, durante o processo de torra de café, os açúcares reagem formando compostos coloridos desejáveis, responsáveis pela cor marrom e características de aroma (SILVA et al., 2014).

Assim, o objetivo da pesquisa é analisar a variabilidade espacial dos valores de brix de café conilon, colhido no estádio cereja, visando a determinação de zonas de manejo em termos de qualidade.

geograficamente na latitude sul $20^{\circ} 31^{\prime}$ 21,44 " e longitude oeste $47^{\circ} 31$ ' 23,16 ". É caracterizado por baixos planaltos e 
colinas, com altitude média de aproximadamente $900 \mathrm{~m}, 3 \% \mathrm{de}$ declividade, predominantemente Latossolo Vermelho. A propriedade consistiu no cultivo com café no estádio cereja (maturação ideal do fruto), da espécie conilon (Coffea canephora), variedade Vitória - "Incaper 8142", de aproximadamente 7 anos e o espaçamento é de 3,0 $\mathrm{m}$ entre linhas e $1,0 \mathrm{~m}$ entre plantas.

A leitura da concentração de sólidos solúveis foi conduzida com um refratômetro portátil, modelo Brix/RICheck, marca Reichert ${ }^{\circledR}$, avaliando 30 plantas por hectare. As amostras foram processadas no Laboratório de Máquinas Agrícolas e Agricultura de Precisão LAMAP do Departamento de Engenharia de Biossistemas (ZEB) da Faculdade de Zootecnia e Engenharia de Alimentos FZEA/USP.

Os resultados foram submetidos a análise de agrupamento utilizando os métodos dendrograma e " $\mathrm{K}$ means", realizado por meio do programa computacional STATISTICA, versão 13.0. A partir da observação de ocorrência da maior transição da distância euclidiana dos

\section{RESULTADO E DISCUSSÃO}

A partir da análise da distribuição dos valores de graus brix presente no histograma da Figura 1, pode-se concluir que ao nível de significância de 5\%, as amostras obtiveram valores não significativos pelo teste de KolmogorofSmirnoff, sendo portando distribuídos de agrupamentos formados, definiu-se o ponto de corte que indica a menor variância e a maior entre os grupos(dados homogêneos entre si). Possibilitando, assim, o número de classes a serem utilizadas para catalogar os valores de graus brix.

Em seguida, conforme o número de classes pré-estabelecido, realiza-se a seleção dos dados pertencentes a cada grupo de valores de graus brix. Se utiliza a análise de agrupamento "K means". Nessa metodologia, são designados os pontos médios de cada grupo, denominados $\mathrm{k}$ centroides. E, logo após, é realizada a atribuição do conjunto de dados ao $\mathrm{k}$ centroide de valor mais próximo. Terminado o processo, posteriormente a alocação de todos os dados, o centroide de cada grupo é recalculado, dando início à próxima iteração. O método é repetido até o momento em que os $\mathrm{k}$ centroides não se modifiquem mais.

Com resultado do método, foi possível estabelecer os valores limites para cada classe de brix: classe 1 que abrange valores inferiores ou iguais a 16,5, classe 2 valores entre 16,6 e 19,7 e classe 3 valores superiores a 19,8 .

forma gaussiana. Também é possível notar a existência de variabilidade das concentrações em brix das 60 amostras, os quais possuem valor médio de 17,45, desvio padrão de 5,19 e são limitados entre 13,54 e 26,66 . 


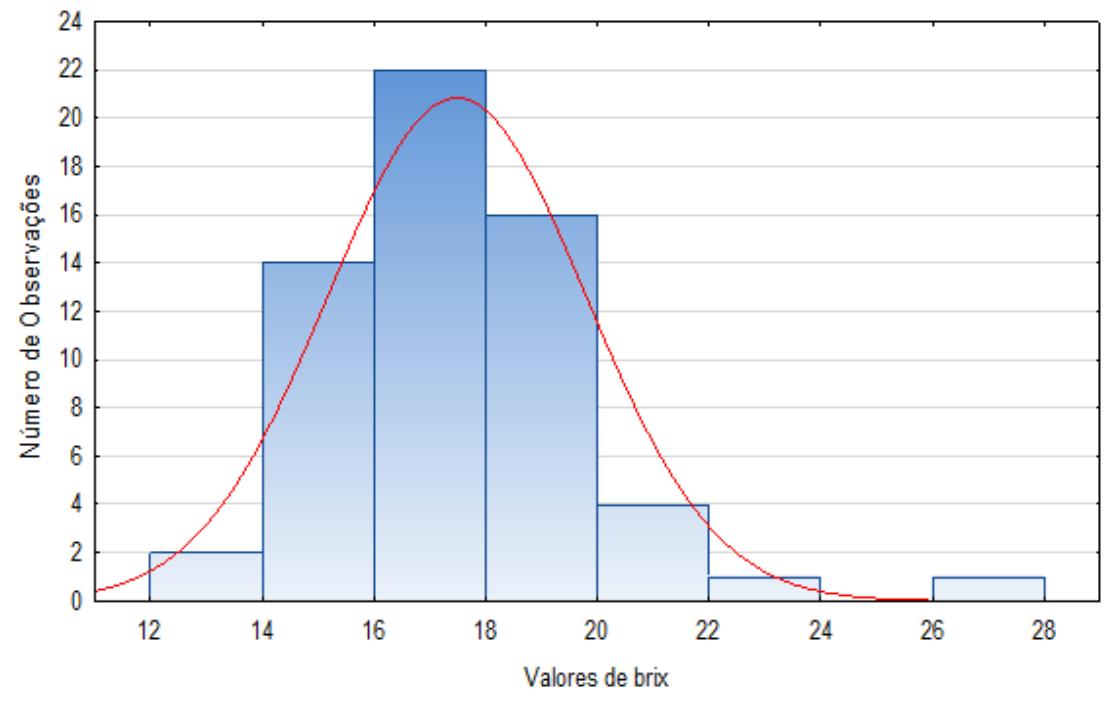

Figura 1. Histograma dos valores de graus brix dos frutos amostrados de café cereja.

A Figura 2 apresenta a distribuição das concentrações de sólidos solúveis, ou seja, o volume de elementos relacionados a um determinado grau brix. A mediana do conjunto de dados é 17,45 e a maioria dos valores de brix encontrados para as amostras estão concentrados entre 15,89 e
18,35. Cenário esse que corrobora com o ilustrado a priori pelo histograma da Figura 1: um bloco volumoso e homogêneo de dados concentrados próximo à mediana em relação ao restante dos valores, mais distribuídos em direção aos extremos e em menor quantidade.

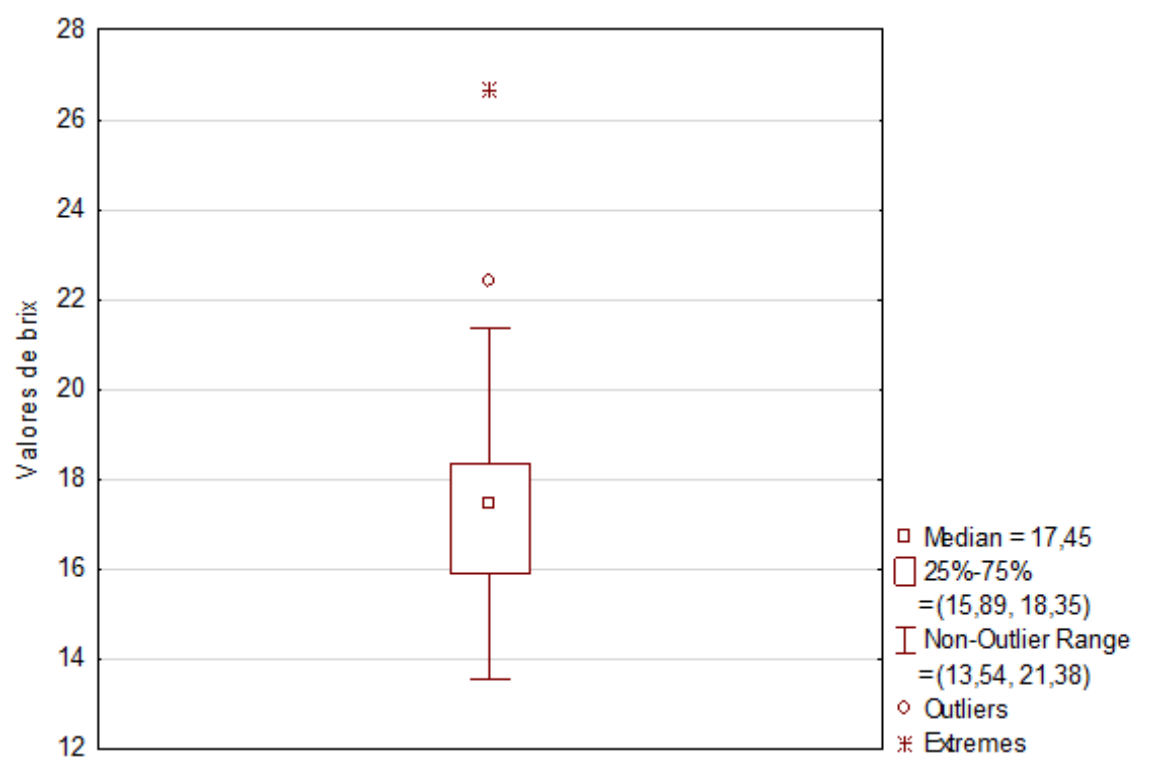

Figura 2. Gráfico de caixa da distribuição dos valores de graus brix dos frutos amostrados de café cereja.

A Figura 3 ilustra o dendrograma da análise de agrupamentos de café. A definição da quantidade de classes baseiase no ponto de corte do dendrograma e fornece subsídios para a aplicação do método "K means". Nesse caso, o ponto onde ocorre o maior salto, no qual há menor variância dentro das classes e menor entre as classes, a distância euclidiana vale 45,78 . 


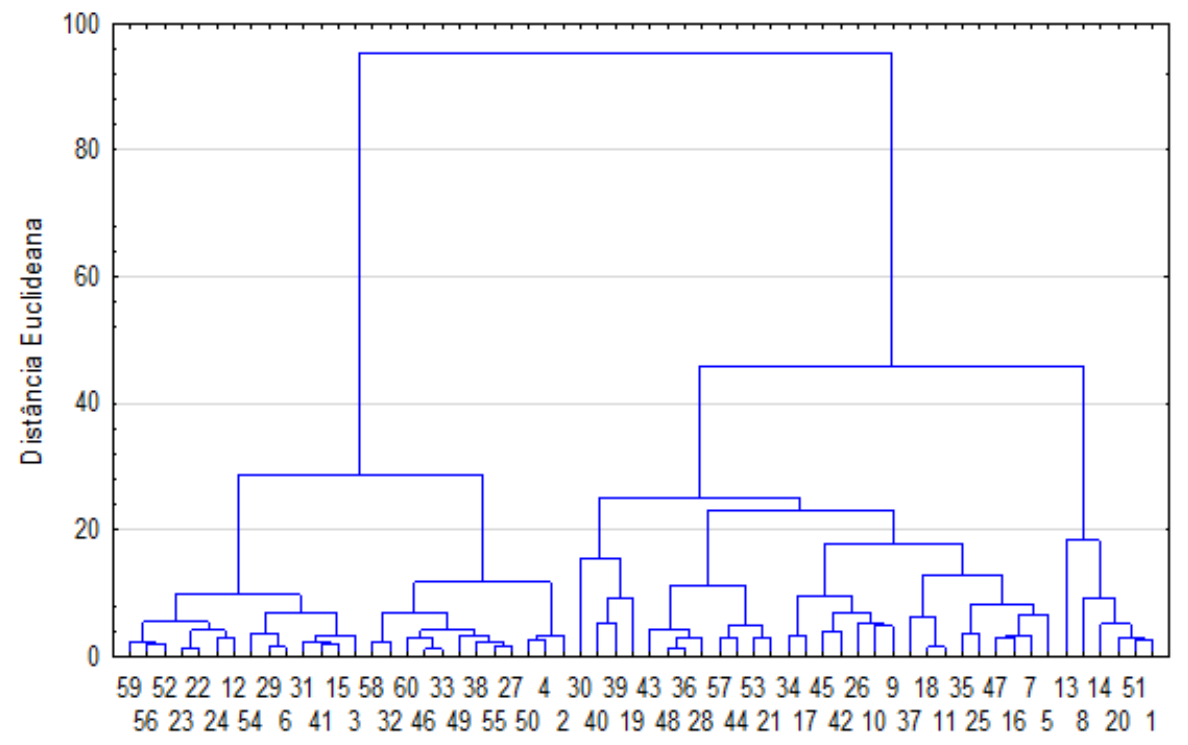

Figura 3. Dendrograma gerado na análise de agrupamento dos valores de brix.

A caixa de dispersão da Figura 4 ilustra a distribuição dos valores de brix nas 3 classes de valores. A classe 1 apresenta 22 componentes com valor médio de 15,3 grau brix; a classe 2 é a mais volumosa com 31 componentes e valor médio de brix de 17,7; enquanto que, a classe 3 é composta por 7 membros, cujo valor médio é de 22,4 grau brix.

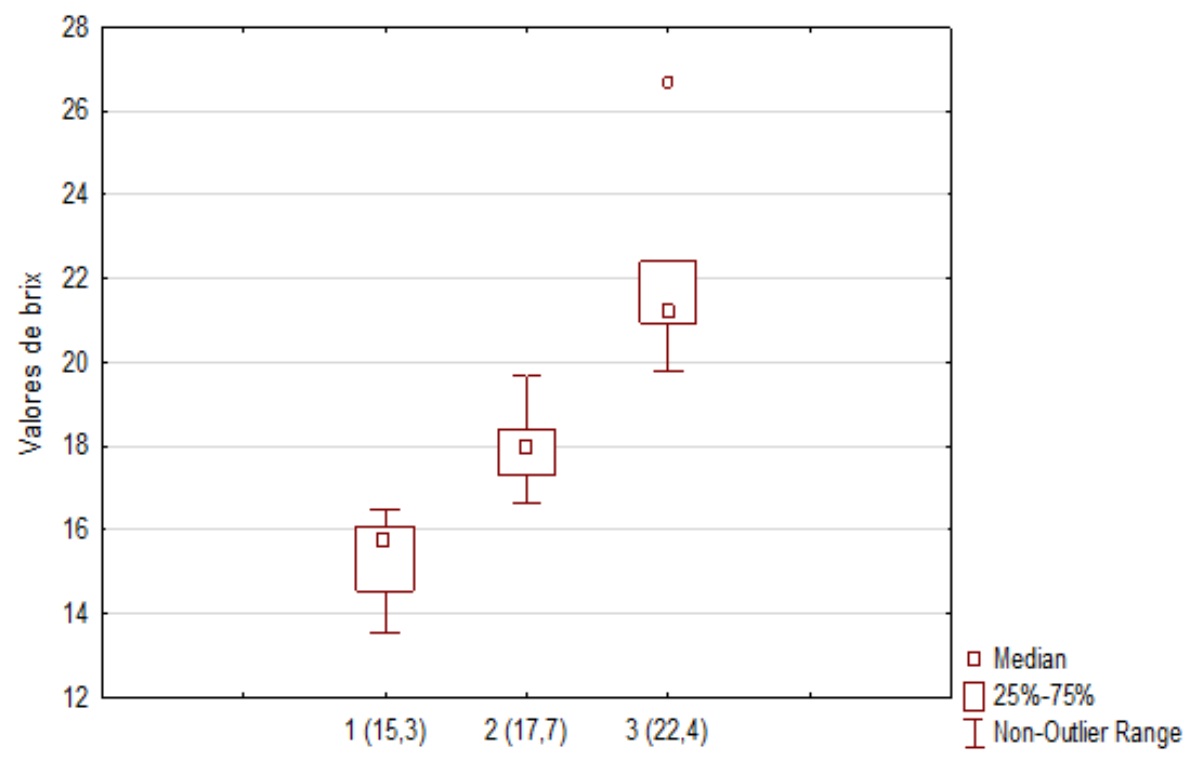

Figura 4. Gráfico de caixa da distribuição dos valores de graus brix nas 3 classes determinadas pela análise de grupamento "K means". 
Observa-se que apenas uma pequena área da propriedade estudada apresentou valor de brix alto. Esse resultado proporcionou concordância ao fato de que a propriedade apresenta características homogêneas (variedade, idade, espaçamento, face de exposição e altitude) quase em sua totalidade, o que propicia condições mais igualitárias para o desenvolvimento da cultura.

$$
\text { Contudo, mesmo que }
$$

discretamente, a existência da variabilidade espacial dos valores de brix se dá pela interação das variáveis ambientais, juntamente com o potencial genético da planta, criando propriedades locais de características distintas. E, sem dúvida, pode determinar uma zona de manejo diferenciada: tanto no âmbito de manejo cultural, utilizando proporções de adubação menores do que nas demais áreas, como também no momento da colheita e pós-colheita, visando a comercialização da saca colhida nessa região de maneira separada.

\section{CONCLUSÕES}

Existe uma variabilidade espacial dos valores de concentração de sólidos solúveis em grau brix dentro de uma plantação de café. Sendo que é possível relacionar essa característica com a qualidade do café desde que haja uma análise posterior que abranja outras variáveis determinantes para a classificação da bebida de café.

Apesar dos valores maiores de brix estarem concentrados em uma pequena área da propriedade, seria interessante implementar uma proposta de manejo

\section{REFERÊNCIAS BIBLIOGRÁFICAS}

ABIC - Associação Brasileira da Indústria de Café. Programa de qualidade do café. Disponível em: 〈http://www.abic.com.br > Acesso em: 10 abr. 2015.
Desta forma, Alves (2009) sugere uma correlação entre o teor de sólidos solúveis da polpa dos frutos maduros com a qualidade da bebida do café. Já que, durante o processo de torra de café, os açúcares reagem formando compostos desejáveis, responsáveis pela cor marrom e as características de aroma. Além disso, conforme indicações da Organização Internacional do Café (1992), valores mais elevados de açúcares podem influenciar numa presença maior de doçura na bebida.

No entanto, ainda não é possível mensurar qual a quantidade de carboidratos presentes na bebida de café que realmente são providos pela condição do fruto, e também o funcionamento detalhado desse mecanismo de transferência que ocorre durante todas as etapas do desenvolvimento da planta. Estabelecendo assim apenas uma relação indireta, no qual as bebidas de maior qualidade derivam de frutos de café com altos valores de brix, ao passo que valores altos de brix não são os únicos fatores que determinam a qualidade da bebida.

diferenciada para essa região. Atitudes como a adequação na proporção de adubação e a separação dos frutos colhidos nesses locais específicos podem tornar a produção mais lucrativa e sustentável.

Além disso, o refratômetro portátil se mostra como uma ferramenta viável para o monitoramento do amadurecimento dos frutos de café e para o estabelecimento de zonas de manejo em relação aos valores de brix.

ALVES, E. A. Variabilidade espacial e temporal da qualidade do café cereja produzido na região das Serras de Minas. Doutorado em Engenharia Agrícola, Viçosa, Brasil: Universidade Federal de Viçosa, 2009. 
CONAB. Companhia Nacional de Abastecimento. Disponível em: <http://www.conab.gov.br/OlalaCMS/uplo ads/arquivos/15_06_10_09_24_57_boletim _cafe_junho_2015.pdf>. Acesso em: $10 \mathrm{de}$ out. 2015.

FERRAZ, G. A. S. et al. Variabilidade espacial da força de desprendimento de frutos do cafeeiro. Engenharia Agrícola. Jaboticabal, SP, v. 34, n. 6, p. 1210-1223, nov./dez. 2014. Disponível em: < http://www.scielo.br/pdf/eagri/v34n6/a16v 34n6.pdf >. Acesso em: 20 de abr. 2015.

Organizacion Internacional Del Café. El despulpado del café por medoi de desmucilaginadoras mecânicas sin processo de fermentación y su efecto em la calidad de bebida de café producido em la región de Apucarana em el estado del Paraná em Brasil: Londres, 1992. n. p. (Reporte Evaluación Sensorial).

QUEIROZ, D. M. et al. Uso de técnicas de agricultura de precisão para a cafeicultura de montanha. In: ZAMBOLIM L. (Ed.) Efeitos da irrigação sobre a qualidade e produtividade do café. Viçosa, MG, p. 77-108, 2004.

SILVA, S. A.; LIMA, J. S. S. Spatial estimation of foliar phosphorus in different species of the genus coffea based on soil properties. Revista Brasileira de Ciência do Solo. Viçosa, MG, v. 38, n. 5, p. 14391447, jun. 2014. Disponível em: <http://www.scielo.br/pdf/rbcs/v38n5/a09v 38n5.pdf $>$. Acesso em: 25 de abr. 2015.

SILVA, S. de A. et al. Characterization and delimitation of the terroir coffee in plantations in the municipal district of Araponga, Minas Gerais, Brazil. Rev. Ciênc. Agron., [s.1.], v. 45, n. 1, p.18-26, 2014. FapUNIFESP (SciELO). DOI: 10.1590/s1806-66902014000100003.

Disponível em: $<$ http://www.scielo.br/scielo.php?script=sc i_arttext\&pid=S1806-
$66902014000100003 \& \operatorname{lng}=\mathrm{en} \& \mathrm{nrm}=\mathrm{iso} \& \mathrm{t}$ $\operatorname{lng}=\mathrm{en}>$. Acesso em: 15 nov. 2015. 